\title{
Day-ahead solar irradiance forecast using sequence-to-sequence model with attention mechanism
}

\author{
Sowkarthika Subramanian ${ }^{1}$, Yasoda Kailasa Gounder ${ }^{1}$, Sumathi Linganathan ${ }^{2}$ \\ ${ }^{1}$ Department of Electrical and Electronics Engineering, Government College of Technology, Coimbatore, India \\ ${ }^{2}$ Department of Computer Science and Engineering, Government College of Technology, Coimbatore, India
}

\section{Article Info}

Article history:

Received Jul 18, 2021

Revised Nov 22, 2021

Accepted Dec 9, 2021

\section{Keywords:}

Attention

Long short-term memory

Sequence-to-sequence LSTM

Solar irradiance forecast

\begin{abstract}
The increasing integration of distributed energy resources (DERs) into power grid makes it significant to forecast solar irradiance for power system planning. With the advent of deep learning techniques, it is possible to forecast solar irradiance accurately for a longer time. In this paper, day-ahead solar irradiance is forecasted using encoder-decoder sequence-to-sequence models with attention mechanism. This study formulates the problem as structured multivariate forecasting and comprehensive experiments are made with the data collected from National Solar Radiation Database (NSRDB). Two error metrics are adopted to measure the errors of encoder-decoder sequence-to-sequence model and compared with smart persistence (SP), back propagation neural network (BPNN), recurrent neural network (RNN), long short term memory (LSTM) and encoder-decoder sequence-to-sequence LSTM with attention mechanism (Enc-Dec-LSTM). Compared with SP, BPNN and RNN, Enc-Dec-LSTM is more accurate and has reduced forecast error of $31.1 \%, 19.3 \%$ and $8.5 \%$ respectively for day-ahead solar irradiance forecast with $31.07 \%$ as forecast skill.
\end{abstract}

This is an open access article under the CC BY-SA license.

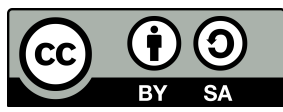

\section{Corresponding Author:}

Sowkarthika Subramanian

Department of Electrical and Electronics Engineering, Government College of Technology

Coimbatore, India

Email: sowkarthika@gct.ac.in

\section{INTRODUCTION}

Integration of solar electricity known as distributed energy resources (DERs) into power grid has gained a rapid development in recent years due to reduction in manufacturing cost and increased efficiency of photovoltaic (PV) panels. The amount of electricity that can be generated from DERs is always a stochastic in nature because of its dependency on weather parameters. This further leads to a challenge for grid operators in estimating generation, distribution and scheduling of power generation. Therefore, an accurate day-ahead forecast of solar irradiance with big data and deep learning model solves this problem.

Forecast models in literature for solar irradiance are persistence model, physical model and statistical model. Very short-term forecast (seconds to less than 30 minutes) is popularly predicted with persistence model [1], [2]. As accuracy of persistence model decreases with increase in forecast horizon, it is not preferred for 24 hours day-ahead forecast. In physical model or numerical weather prediction models [1], [3], the state of the atmosphere is described by mathematical equations which require numerical methods to solve. Forecast employed with physical model leads to erroneous result for sudden change in values of meterological variables such as relative humidity, wind speed and wind direction. Artificial neural network (ANN) based multilayer perceptron model [4], [5] with Levenberg-Marquardt algorithm was proposed to forecast 24 hours ahead solar 
irradiance and found that the usage of meterological parameters as input variables gives more accuracy in forecast. Input variables with higher dimension [6]-[9] (up to 900 inputs) are used with ANN models of different architecture to predict short term global solar irradiance of $20 \%$ reduction in errors. Deep learning models are the subset of machine learning and these models on solar irradiance forecast results with higher accuracy comapared to machine learning models. A method of day-ahead solar irradiance forecast using long short-term memory (LSTM) network with weather variables as feature vectors was developed [10] and results prove that LSTM outperforms all the other conventional forecast methods in terms of forecast accuracy. Jeon et al. [11] proposed an LSTM based deep learning model for solar irradiance forecast with weather variables and also solar irradiance of the previous day as feature vectors. Simulation result shows the improvement in forecast accuracy if solar irradiance of previous day is also used as input feature. Gao et al. [12] proposed gated recurrent unit (GRU) based model for hourly day-ahead solar irradiance forecast using weather variables.

In this paper, studies are made to forecast day-ahead solar irradiance using LSTM based encoder- decoder models with attention mechanism. Intially, datas are cleaned and converted into structured multivariate problem to train with encoder-decoder sequence-to-sequence models. Based on pearson correlation coefficient, input variables are selected from the list of meteorological parameters. Comprehensive experiments are made to determine the forecast accuracy considering meterological parameters as input variable. Experiments have shown that LSTM based encoder-decoder sequence-to-sequence models with attention mechanism have reduced errors comparatively. Forecast horizon [14] from the perspective of decision making activity in microgrid or smartgrid are classified as very short-term forecast, short-term forecast, medium-term forecast and long-term forecast. Very short-term forecast is used in real time monitoring of photovoltaic power and the forecast horizon is from few seconds to minutes ahead. Short-term forecast is used in decsion making applications involved in power system operation such as economic dispatch, unit commitment. Forecast horizon for short-term forecast is up to 48 to 72 hours ahead. Schedule and maintenance of power plant are planned with medium-term forecast and its horizon is upto one week ahead. Long term forecast helps in the assessment of solar energy and its horizon is from months to years. Unit commitment [15], [16] for power plants such as biomass, nuclear, and coal, are one day-ahead and for power plants such as gas and oil are hour ahead. This time horizon is formulated depending on their startup and shutdown times. In such a case with renewable integration into grid, unit commitment and economic dispatch decisions vary depending on solar forecasts.

In this paper, day-ahead solar irradiance is forecasted using different deep-learning techniques. In dayahead forecast previous day's data is used as input to forecast irradiance of next 11 hours with a resolution of one hour. In general, geographical locations [17] also determine the forecast error and hence the models described here are tested for different locations with different climatic conditions also. This paper is organised as follows: methodology is described in section 2, description of data and preprocessing in section 3 , experiments and results in section 4 and conclusion and future work in section 5 .

\section{METHOD}

Long short term memory network is base for all the other models. Hence, architecture of LSTM and LSTM based encoder-decoder sequence-to-sequence models with attention mechanism and a benchmark algorithm are described in detail. Under benchmark algorithm, smart persistence is used to compare the proposed method.

\subsection{Smart persistence-benchmark algorithm}

Forecast error varies with dataset, location and horizon. Hence for a good comparison, benchmarking algorithm such as smart persistence model (SP) [18] or scaled persistence model is suggested. Smart persistence model suggest that the predicted value at the next moment $\hat{G}(t+h)$ is the product of clear-sky index $k_{c s}(t)$ and clear-sky irradiance at next moment $G_{c s}(t+h)$.

$$
\begin{gathered}
k_{c s}(t)=\frac{G(t)}{G_{c s}(t)} \\
\hat{G}(t+h)=k_{c s}(t) G_{c s}(t+h)
\end{gathered}
$$

where $k_{c s}(t)$ is the clear-sky index,$G_{c s}(t)$ is the clear-sky irradiance and h in 2 is the forecast horizon. 


\subsection{Encoder-decoder sequence-to-sequence architecture}

Traditional neural network like back propagation neural network (BPNN), do not have memory to understand and process sequential data. This was overcome by recurrent neural network (RNN) algorithm [19]. RNNs have loops within them and makes the informations to persist. However RNN suffers from vanishing and exploding gradient problems that prevents it from learning large sequences. Hochreiter et al. proposed LSTM [20] network that can process sequential data effectively with recurrent neural network as shown in Figure 1 .

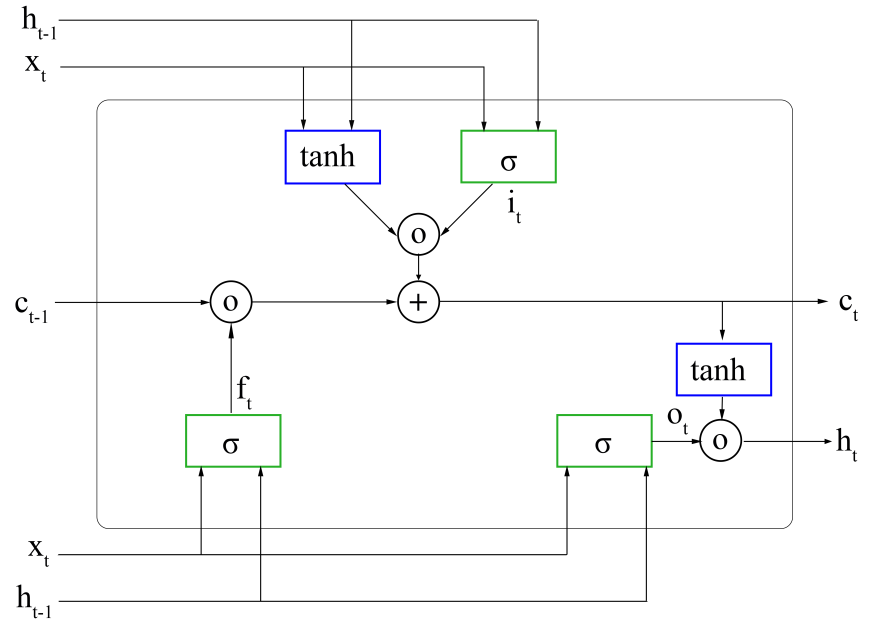

Figure 1. LSTM cell structure

Input variables of a single LSTM units are current time step input vector $X_{t}$, output of the previous LSTM unit $h_{t-1}$ and memory of the previous LSTM unit also called cell state $c_{t-1}$. The outputs of a single LSTM unit are output of the hidden layer $h_{t}$ and memory at the current time step $c_{t}$. Each LSTM unit processes the information through forget gate $\left(f_{t}\right)$, input gate $\left(i_{t}\right)$ and output gate $\left(o_{t}\right)$ according to (3), (4) and (5).

$$
\begin{gathered}
f_{t}=\sigma\left(W_{x f o r} x_{t}+W_{h f o r} h_{t-1}+b_{\text {for }}\right) \\
i_{t}=\sigma\left(W_{\text {xinp }} x_{t}+W_{\text {hinp }} h_{t-1}+b_{\text {inp }}\right) \\
o_{t}=\sigma\left(W_{\text {xout }} x_{t}+W_{\text {hout }} h_{t-1}+b_{\text {out }}\right)
\end{gathered}
$$

Where $W_{x f o r}, W_{h f o r}$ are forget gate's weight matrix, $W_{x i n p}, W_{\text {hinp }}$ are input gate's weight matrix and $W_{\text {xout }}, W_{\text {hout }}$ are output gate's weight matrix; $b_{f o r}, b_{\text {inp }}$ and $b_{\text {out }}$ are bias values of forget gate, input gate and output gate respectively. $\sigma$ represents sigmoid activation function. Forget gate $\left(f_{t}\right)$ decides, which part of the informations are to be erased and which part of the informations are to be retained and outputs a number between 0 and 1 through sigmoid function. Input gate $\left(i_{t}\right)$ and forget gate $\left(f_{t}\right)$ specifies the part of the informations to be added with the cell state. Finally, output gate $\left(o_{t}\right)$ decides the information output from cell state. Cell state $c_{t}$ and current output of hidden layer are calculated by (6) and (7),

$$
\begin{gathered}
c_{t}=f_{t} \odot c_{t-1}+i_{t} \odot \tanh \left(W_{x c e l l} x_{t}+W_{h c e l l} h_{t-1}+b_{\text {cell }}\right) \\
h_{t}=o_{t} \odot \tanh \left(c_{t}\right)
\end{gathered}
$$

where $\odot$ represents the hadamard product that performs element-wise matrix multiplication.

Encoder-decoder sequence-to-sequence architecture uses LSTM (Enc-Dec-LSTM) as encoder component, Luong's attention layer, another LSTM network as decoder component and a dense layer as shown in Figure 2. Encoder-decoder sequence-to-sequence architecture although developed for natural language translation, it had been succesfully applied for time-series forecasting [21] such as air-quality, and traffic prediction. Encoder [22] encodes the information from input into a fixed length vector. The final outputs of the encoder are 
discarded and the internal state and hidden state combinedly called fixed length vector is fed into the decoder. Decoder is also given previous hour of the target and trained to predict next hour. This process of training is called teacher-forcing.

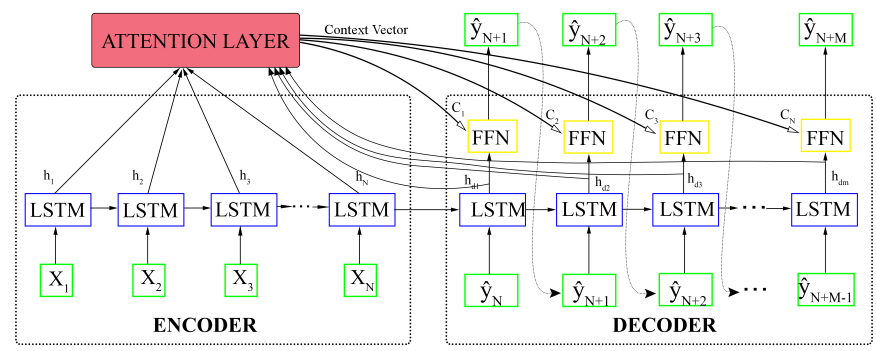

Figure 2. Encoder-decoder sequence-to-sequence architecture

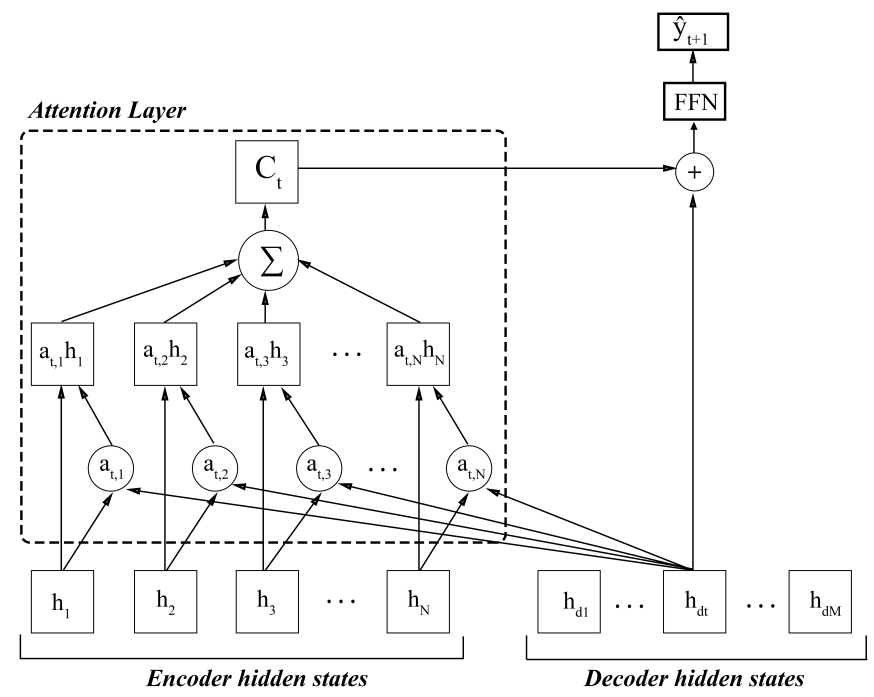

Figure 3. Attention layer

\subsection{Attention mechanism}

According to Luong et al. [23] the potential issue of the encoder is, by compressing all necessary information of input into a fixed-length vector may fail to generate long sequence from the decoder. Attention layer as shown in Figure 3 allows the model to access all the past hidden states of encoder instead of the last hidden layer alone. The alignment score $e_{t, i}$ for Luong's attention is calculated as in (8),

$$
\begin{gathered}
e_{t, i}=h_{d t}^{T} \cdot h_{i} \\
a_{t, i}=\frac{\exp \left(e_{t, i}\right)}{\sum_{j=1}^{N} \exp \left(e_{t, j}\right)} \\
C_{t}=\sum_{i=1}^{N} a_{t, i} h_{i}
\end{gathered}
$$

where $h_{d t}$ is current target state or $t^{t h}$ hidden state of decoder and $h_{i}$ is $i^{\text {th }}$ hidden state of encoder. Attention weight $a_{t, i}$ as in 9 is calculated by softmaxing the alignment score to sum up to 1 . Context vector as in $(10)$ is computed by element-wise multiplication of ith hidden state of encoder and attention weight. The context vector is then concatenated with current target state $h_{d t}$ and is fed into a fully connected feed-forward network 
(FFN). Computation time [10], [12] for these networks are not critical as training is offline but the forecast using trained network is fast.

\section{DESCRIPTION OF DATA AND PREPROCESSING}

\subsection{Dataset}

Solar irradiance data can be obtained either from a measuring instrument installed at site or through satellite derived irradiance dataset. Though satellite derived dataset is less accurate compared to a dataset collected from a measuring instrument, satellite derived dataset is often used by researchers [24], [25] because of its open access, ease of use, wide temporal and spatial coverage and almost no data is missed. The data set containing real-world meterological values are collected from the National Renewable Energy Laboratory's (NREL), National Solar Radiation Database (NSRDB) [26] for New Delhi, India. Hourly data of global horizontal irradiance (GHI), temperature, pressure, relative humidity, wind direction and wind speed are obtained from the year 2009 to 2015. Solar irradiance exists only during daytime and hence the hours between 7:00 AM and 5:00 PM are considered. After analysing the dataset, solar irradiance peaks in the month of April and May comparatively for selected location and this shows its seasonal behaviour.

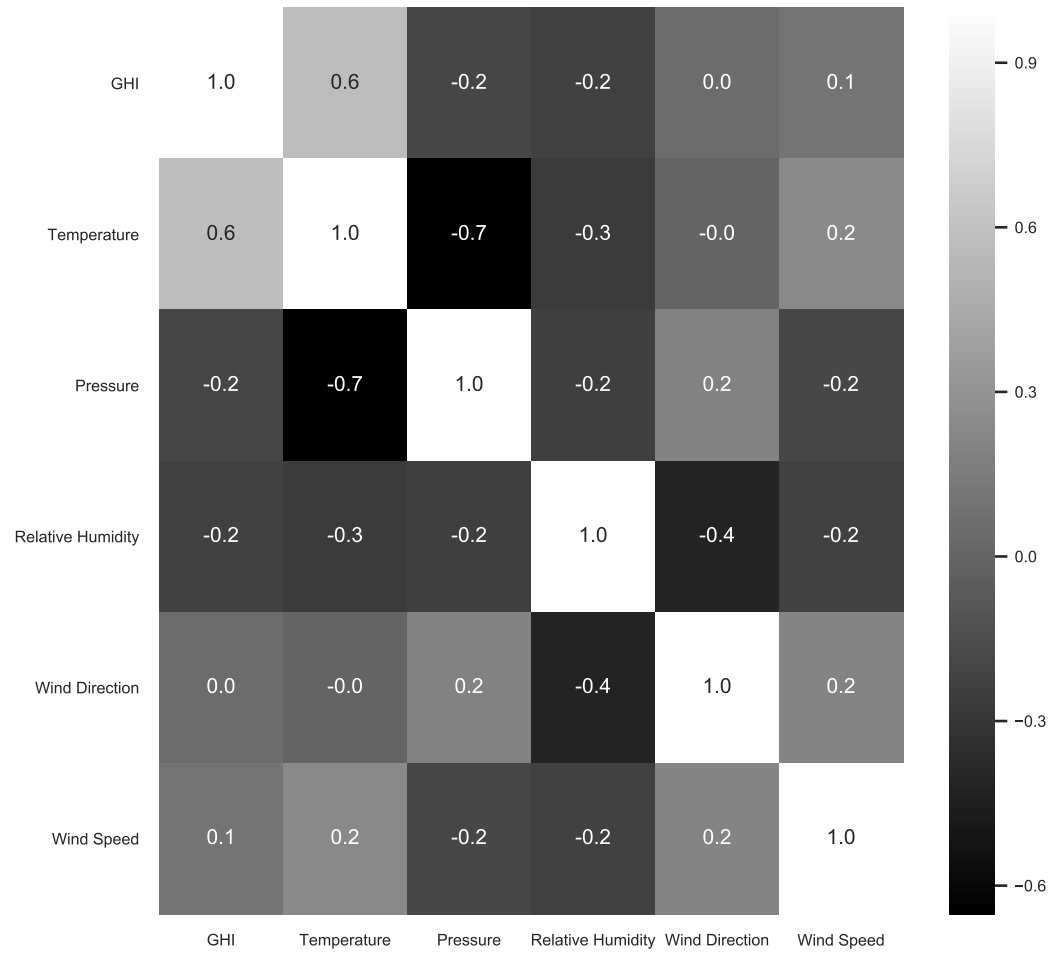

Figure 4. Heat map with correlation coefficient between input variables

\subsection{Data normalization}

The datas loaded into neural network are normalized in the range of $[0,1]$. According to (11) $d_{i}$ is the data before normalization, $d_{i}^{*}$ is the data after normalization, $d_{\min }$ and $d_{\max }$ are the minimum and maximum value of the variable. The aim of data normalization is to convert the numeric values in dataset to a common value.

$$
d_{i}^{*}=\frac{d_{i}-d_{\min }}{d_{\max }-d_{\min }}
$$

\subsection{Correlation}

Linear relationship between two variables are measured commonly with the Pearson's correlation coefficient. Correlation between solar irradiance and other weather variables are measured using Pearson's 
correlation coefficient as shown in Figure 4 From the analyses of Pearson's correlation coefficient, temperature is found to be positively correlated with GHI and relative humidity is found to be negatively correlated with GHI. In literature, Evans [27] and Denes et al. [28] classified the absolute value for correlation factor as very weak if value is between 0 and 0.19 , weak if value is between 0.20 and 0.39 , moderate if value is between 0.40 and 0.59 , strong if value is between 0.6 and 0.79 and very strong if value is between 0.8 and 0.99 . As per the above classification wind direction and wind speed can be neglected as their correlation is very weak with GHI. Sliding window technique is used in preprocessing of data.

\section{EXPERIMENTS AND RESULTS}

\subsection{Training and testing data}

The data from January 2009 to December 2013 are taken as training set and the data from 2014 are taken as test set. Training and validation data are split using test train splitter which devotes $80 \%$ of data to train and remaining $20 \%$ of data for validation. Input data with weather variables are in different range of values. Hence datasets are rescaled to lie in the range of $[0,1]$ and it is called normalization of datasets. Datasets are normalized using MinMaxScaler in scikit-learn according to (11).

\subsection{Metrics}

Standard statistical measures such as root mean square error (RMSE), and mean absolute error (MAE) are commonly used to measure the accuracy of forecast model [29],

$$
\begin{aligned}
R M S E & =\sqrt{\frac{1}{n} \sum_{i=1}^{n}\left(Y_{\text {pred }, i}-Y_{\text {actual }, i}\right)^{2}} \\
M A E & =\frac{1}{n} \sum_{i=1}^{i=n}\left|Y_{\text {pred }, i}-Y_{\text {actual }, i}\right|
\end{aligned}
$$

where $Y_{\text {pred }}$ is the predicted irradiance value and $Y_{\text {actual }}$ is the actual irradiance value. To have a good comparision, forecast skill (FS) [18] is one of the most recommended metric in the world of forecast, where SP in 14 is smart persistence.

$$
\text { ForecastSkill }=1-\frac{R M S E_{\text {proposed }}}{R M S E_{S P}}
$$

\subsection{Experiments}

Experiments described here uses Keras version 2.3.1 to implement BPNN, RNN, LSTM and LSTM based encoder-decoder sequence-to-sequence model with attention. Hyper-parameter for the above models are tuned based on grid-search method. BPNN has 55 units and 95 units in hidden layer1 and hidden layer2 respectively whereas RNN has 95 units and 105 units, LSTM has 85 units and 125 units in their repective hidden layer1 and hidden layer2. Encoder and decoder layer has each 95 units in Enc-Dec-LSTM network. Dropout of 0.2 is used in each of input layers as a regularisation technique. Adam optimiser is used for optimization as it combines the best features of RMSprop and AdaGrad and batch size is set as 100 from grid-search method. Smart persistence model is free of training and tuning of parameters.

\subsubsection{Forecast results}

Forecast is performed with temperature, relative humidity and pressure as input variables and EncDec-LSTM model is compared with LSTM, RNN, BPNN and SP. Clearsky GHI is used in smart persistence to forecast day-ahead irradiance. The hourly input variables from 8:00 am to 5:00 pm are considered and therefore for a day, 10 timesteps are accounted. Different lagging time from 10 hour to 22 hour are tested and found the model results with least error for a 10 hour lagging time. Thus for day-ahead forecast, previous day's 10 hours of data is given as input to predict next day's 10 hours of solar irradiance with a resolution of one hour. 


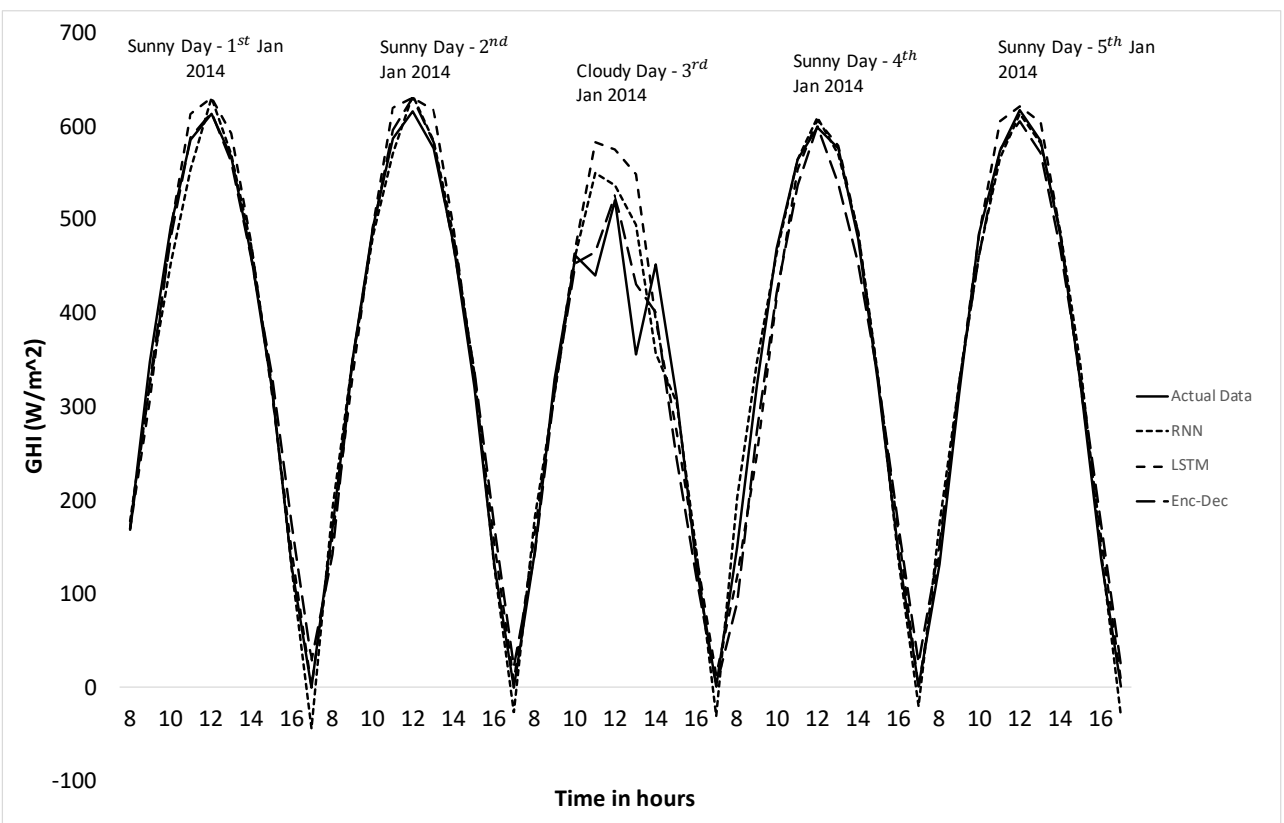

Figure 5. Comparision of hourly forecasted irradiance values

Table 1. Performance comparision of different algorithms

\begin{tabular}{lccc}
\hline Algorithm & RMSE $\left(W / m^{2}\right)$ & MAE $\left(W / m^{2}\right)$ & FS $(\%)$ \\
\hline Enc-Dec-LSTM & 100.57 & 60.27 & 31.07 \\
LSTM & 104.52 & 61.88 & 28.37 \\
RNN & 109.95 & 64.37 & 24.64 \\
BPNN & 124.67 & 104.13 & 14.56 \\
SP & 145.91 & 79.77 & 0 \\
\hline
\end{tabular}

Forecast results in terms of error metric are shown in Table 1. Enc-Dec-LSTM outperforms the other models and compared to SP, BPNN and RNN, RMSE is reduced by $31.1 \%, 19.3 \%, 8.5 \%$ respectively and MAE is reduced by $24.4 \%, 42.1 \%, 6.4 \%$ respectively. Less forecast skill indicates that the models performance is almost same as that of smart persistence model. Enc-Dec-LSTM model has the highest forecast skill of $31.07 \%$ which indicates that the model performs better than any other model compared here. As shown in Figure 5 , Enc-Dec-LSTM model's forecast is nearer to actual data even on a cloudy day and hence its overall error is less compared to the other models.

Average monthly RMSE of the test dataset is shown in Table 2 and its seen that the error peaks during Monsoon season. As shown in Figure 4, GHI is highly correlated with temperature variable and thus the monthly correlation of temperature with GHI is tested on the test dataset. The correlation of temperature with GHI during the months July, August and September are low which results with highest error during Monsoon season.

Table 2. Average monthly RMSE $\left(W / m^{2}\right)$ and MAE $\left(W / m^{2}\right)$ of test dataset

\begin{tabular}{llcccccccccccc}
\hline Error & Algorithm & Jan & Feb & Mar & Apr & May & Jun & Jul & Aug & Sept & Oct & Nov & Dec \\
\hline RMSE & Enc-Dec & \multirow{2}{*}{1.41} & 127.82 & 121.57 & 81.42 & 96.69 & 105.02 & $\mathbf{1 3 1 . 4 5}$ & $\mathbf{1 4 3 . 6 3}$ & 102.66 & 48.68 & 24.83 & 57.46 \\
& -LSTM & & & & & & & & & & & \\
& LSTM & 98.95 & 137.23 & 124.82 & 80.61 & 99.17 & 104.74 & $\mathbf{1 3 6 . 1 1}$ & $\mathbf{1 4 5 . 9 8}$ & 106.86 & 54.82 & 24.06 & 68.41 \\
& RNN & 123.18 & 155.47 & 130.17 & 82.13 & 98.71 & 102.13 & $\mathbf{1 3 8 . 4 3}$ & $\mathbf{1 5 3 . 2 9}$ & 108.72 & 51.37 & 24.63 & 65.19 \\
& BPNN & 98.76 & 133.35 & 136.35 & 114.75 & 121.88 & 114.53 & $\mathbf{1 3 7 . 2 4}$ & $\mathbf{1 5 3 . 9 8}$ & 123.97 & 73.09 & 49.03 & 64.87 \\
MAE & Enc-Dec & & & & & & & & & & & \\
& -LSTM & 59.52 & 86.20 & 79.86 & 50.87 & 56.02 & 58.64 & $\mathbf{8 8 . 2 1}$ & $\mathbf{9 5 . 9 5}$ & 63.06 & 30.32 & 19.23 & 34.70 \\
& LSTM & 60.85 & 85.63 & 76.18 & 47.38 & 59.34 & 64.02 & $\mathbf{9 3 . 3 1}$ & $\mathbf{9 6 . 0 5}$ & 67.22 & 36.06 & 17.89 & 37.76 \\
& RNN & 69.90 & 92.07 & 80.70 & 52.60 & 61.95 & 62.73 & $\mathbf{9 3 . 0 5}$ & $\mathbf{1 0 1 . 0 6}$ & 70.23 & 32.22 & 18.21 & 36.97 \\
& BPNN & 71.29 & 103.27 & 106.79 & 99.72 & 105.91 & 93.45 & $\mathbf{1 0 8 . 8 5}$ & $\mathbf{1 2 2 . 9 2}$ & 97.72 & 59.49 & 39.92 & 47.15 \\
\hline
\end{tabular}




\subsubsection{Forecast results at different location}

In addition, the geographical location and climatic conditions also determine the forecast accuracy and hence a test is made on three different location with different climatic conditions to study and compare the feasibility of Enc-Dec-LSTM model. LSTM and Enc-Dec-LSTM models are compared for the datasets collected from NSRDB at different locations for different climatic conditions according to Koppen-Geiger climate classification. The data from year 2009 to 2013 are set as training dataset and 2014 as testing dataset. Table 3 lists the day-ahead RMSE of LSTM and Enc-Dec-LSTM in which Enc-Dec-LSTM has least error in all different locations with different climatic conditions. According to Koppen-Geiger climate classification Csa, Bsh and Aw as listed in Table 3 denotes hot-summer mediterranean climate, hot semi-arid (steppe) climate, tropical savanna wet climate respectively.

Table 3. Day-ahead RMSE of forecast model at different locations

\begin{tabular}{lcccc}
\hline Latitude & Longitude & Climate & LSTM RMSE $\left(W / m^{2}\right)$ & Enc-Dec-LSTM RMSE $\left(W / m^{2}\right)$ \\
\hline 23.25 & 77.35 & Csa & 101.42 & 98.47 \\
26.25 & 73.05 & Bsh & 88.44 & 85.5 \\
22.65 & 88.45 & Aw & 121.46 & 117.55 \\
\hline
\end{tabular}

\subsubsection{Comparision with recently published papers}

A comparision of recently published works in one day-ahead solar irradiance forecast is made in Table 4 Emerging deep learning techniques shows great improvement in accuracy for day-ahead solar irradiance forecast. Forecast error can also depend on geographical location and climatic condition and therefore forecast skill as developed by Yang [18] can be the best reference to compare with other models. As per forecast skill comparision in Table 4. LSTM based encoder-decoder sequence-to-sequence with attention mechanism has highest skill of $31.07 \%$ and thus it outperforms the other models.

Table 4. Comparision of day-ahead solar irradiance with recently published works

\begin{tabular}{|c|c|c|c|c|}
\hline Author & Algorithm & Location & RMSE & $\mathrm{FS}(\%)$ \\
\hline Larson et al. 30 & $\mathrm{LSO}^{a}$ and $\mathrm{NWP}^{b}$ & San Diego, USA & $27.5 \%$ & 24 \\
\hline Aryaputera et al. 31 & $\mathrm{WRF}^{c}$ and $\mathrm{ETS}^{d}$ & Station 500, Singapore & $188\left(W / m^{2}\right)$ & 12.9 as per 14 \\
\hline Hai et al. 32] & $\mathrm{DFT}^{e}$ & Qingdao, China & $127.3\left(\mathrm{~W} / \mathrm{m}^{2}\right)$ & 6.3 \\
\hline Qing and Niu et al.10 & LSTM & Cape Verde, Santiago & $122.72\left(\mathrm{~W} / \mathrm{m}^{2}\right)$ & - \\
\hline Gao et al. 12 & GRU & Denver, USA & $122.45\left(W / m^{2}\right)$ & 28.4 \\
\hline Present work & Enc-Dec-LSTM & New Delhi, India & $100.57\left(\mathrm{~W} / \mathrm{m}^{2}\right)$ & 31.07 \\
\hline
\end{tabular}

\section{CONCLUSION AND FUTURE WORK}

This paper attempts to study the encoder-decoder sequence-to-sequence models with attention for solar irradiance forecast which was originally developed for natural language processing. Initially datas are collected from NSRDB site and processed with sliding window technique and then normalised before applying to deep-learning models to improve accuracy. Unwanted features of data are removed using pearson's correlation method. Five years of data are supplied for training and one year of data is supplied for testing. Based on the experimental results, LSTM based encoder-decoder sequence-to-sequence models with attention mechanism outperforms the other techniques as it combines both encoder-decoder facility and attention mechanism which reduces error and improves accuracy, though the computation time of Enc-Dec-LSTM model is higher than LSTM. Further the recently developed CNN based hybrid models and transformer models could also be studied for solar irradiance forecast.

\section{REFERENCES}

[1] P. Mathiesen and J. Kleissl, "Evaluation of numerical weather prediction for intra-day solar forecasting in the continental United States," Solar Energy, vol. 85, no. 5, pp. 967-977, May 2011, doi: 10.1016/j.solener.2011.02.013.

[2] S. Dutta et al., "Load and renewable energy forecasting for a microgrid using persistence technique," Energy Procedia, vol. 143, pp. 617-622, Dec. 2017, doi: 10.1016/j.egypro.2017.12.736.

[3] S. Pelland, G. Galanis, and G. Kallos, "Solar and photovoltaic forecasting through post-processing of the global environmental multiscale numerical weather prediction model," Progress in Photovoltaics: Research and Applications, vol. 21, no. 3, pp. 284-296, Nov. 2011, doi: 10.1002/pip.1180. 
[4] A. Mellit and AM. Pavan,'A 24-h forecast of solar irradiance using artificial neural network: Application for performance prediction of a grid-connected PV plant at Trieste, Italy," Solar Energy, vol. 84, no. 5, pp. 807-821, May. 2010, doi: 10.1016/j.solener.2010.02.006.

[5] H. A. Mohammad, A. Jehad, and Y. Ola, "Solar photovoltaic power forecasting in Jordan using artificial neural networks," International Journal of Electrical and Computer Engineering (IJECE), vol. 8, no. 1, pp. 497-504, Feb. 2018, doi: 10.11591/ijece.v8i1.pp497-504.

[6] R. Meenal, P. A. Michael, D. Pamela, and E. Rajasekaran, "Weather prediction using random forest machine learning model," Indonesian Journal of Electrical Engineering and Computer Science (IJEECS), vol. 22, no. 2, pp. 1208-1215, May 2021, doi: 10.11591/ijeecs.v22.i2.pp1208-1215.

[7] Y. Amellas, O. E. Bakkali, A. Djebil, and A. Echchelh, "Short-term wind speed prediction based on MLP and NARX network models," Indonesian Journal of Electrical Engineering and Computer Science (IJEECS), vol. 18, no. 1, pp. 150-157, April 2020, doi: 10.11591/ijeecs.v18.i1.pp150-157.

[8] F. V. Gutierrez-Corea, M. A. Manso-Callejo, M. P. Moreno-Regidor, and M. T. Manrique-Sancho, "Forecasting short-term solar irradiance based on artificial neural networks and data from neighboring meteorological stations," Solar Energy, vol. 134, pp. 119131, Sept. 2016, doi: 10.1016/j.solener.2016.04.020.

[9] N. Susithra, G. Santhanamari, M. Deepa, P. Reba, K. C. Ramya, and L. Garg, "Deep learning-based activity monitoring for smart environment using radar," In: Challenges and Solutions for Sustainable Smart City Development. EAI Springer Innovations in Communication and Computing. Springer, Cham., May 2021, doi: 10.1007/978-3-030-70183-3_5.

[10] X. Qing and Y. Niu, "Hourly day-ahead solar irradiance prediction using weather forecasts by LSTM," Energy, vol. 148, pp. 461468, April 2018, doi: 10.1016/j.energy.2018.01.177.

[11] B. K. Jeon and E. J. Kim, "Next-Day Prediction of Hourly Solar Irradiance Using Local Weather Forecasts and LSTM Trained with Non-Local Data," Energies, vol. 13, no. 20, Oct. 2020, doi: 10.3390/en13205258.

[12] B. Gao, X. Huang, J. Shi, Y. Tai, and X. Rui, "Predicting day-ahead solar irradiance through gated recurrent unit using weather forecasting data," Journal of Renewable and Sustainable Energy, vol. 11, no. 4, Aug. 2019, doi: 10.1063/1.5110223.

[13] M. Alhussein, S. I. Haider, and, K. Aurangzeb, "Microgrid-level energy management approach based on short-term forecasting of wind speed and solar irradiance," Energies, vol. 12, no. 8, April 2019, doi: 10.3390/en12081487.

[14] A. Sharma and A. Kakkar, "Forecasting daily global solar irradiance generation using machine learning," Renewable and Sustainable Energy Reviews, vol. 82, pp. 2254-2269, Feb. 2018, doi: 10.1016/j.rser.2017.08.066.

[15] J. Zhang et al., "Baseline and target values for regional and point PV power forecasts: Toward improved solar forecasting," Solar Energy, vol. 122, pp. 804-819, 2015, doi: 10.1016/j.solener.2015.09.047.

[16] G. Ganesh, G. V. Kumar, A. R. VijayBabu, G. S. Rao, and Y. R. Tagore, "Performance Analysis and MPPT Control of a Standalone Hybrid Power Generation System”, Journal of Electrical Engineering, vol. 15, no. 1, pp. 334-343, June 2015.

[17] C. Voyant, T. Soubdhan, P. Lauret, M. David, and M. Muselli, "Statistical parameters as a means to a priori assess the accuracy of solar forecasting models," Energy, vol. 90, pp. 671-679, Aug. 2015, doi: 10.1016/j.energy.2015.07.089.

[18] D. Yang, "A guideline to solar forecasting research practice: Reproducible, operational, probabilistic or physically-based, ensemble, and skill (ROPES)," Journal of Renewable and Sustainable Energy, vol. 11, no. 2, April 2019, doi: 10.1063/1.5087462.

[19] A. Tealab, "Time series forecasting using artificial neural networks methodologies: A systematic review," Future Computing and Informatics Journal, vol. 3, no. 2, pp. 334-340, Dec. 2018, doi: 10.1016/j.fcij.2018.10.003.

[20] S. Hochreiter and J. Schmidhuber, "Long short-term memory," Neural Computation, vol. 9, no. 8, pp. 1735-1780, Nov. 1997, doi: 10.1162/neco.1997.9.8.1735.

[21] S. Du, T. Li, Y. Yang, and S. J. Horng, "Multivariate time series forecasting via attention-based encoder-decoder framework," Neurocomputing, vol. 388, pp. 269-279, May 2020, doi: 10.1016/j.neucom.2019.12.118.

[22] I. Sutskever, O. Vinyals, and Q. V. Le, "Sequence to Sequence Learning with Neural Networks," arXiv preprint, Dec. 2014, arxiv: $1409.3215 \mathrm{v} 3$.

[23] M. Luong, H. Pham, and C. D. Manning, "Effective Approaches to Attention-based Neural Machine Translation," arXiv preprint, Sept. 2015, arXiv:1508.04025v5.

[24] D. Yang, "A correct validation of the National Solar Radiation Data Base (NSRDB)," Renewable and Sustainable Energy Reviews, vol. 97, pp. 152-155, Dec. 2018, doi: 10.1016/j.rser.2018.08.023.

[25] G. M. Yagli, D. Yang, and D. Srinivasan, "Automatic hourly solar forecasting using machine learning models," Renewable and Sustainable Energy Reviews, vol. 105, pp. 487-498, May 2019, doi: 10.1016/j.rser.2019.02.006.

[26] NREL. [online]. Available: https://maps.nrel.gov/nsrdb-viewer [accessed December 2020]

[27] J. D. Evans, "Straightforward statistics for the behavioral sciences", Thomson Brooks/Cole Publishing Company, 1996.

[28] K. A. Denes, S. M. Yhan, P. C. Bernardes, and C. A. Conte-Junior, "Relationship between COVID-19 and weather: Case study in a tropical country," International Journal of Hygiene and Environmental Health, vol. 229, Aug. 2020, doi: 10.1016/j.ijheh.2020.113587

[29] R. J. Hyndman and A. B. Koehler, “Another look at measures of forecast accuracy," International Journal of Forecasting, vol. 22, no. 4, pp. 679-688, Dec. 2006, doi: 10.1016/j.ijforecast.2006.03.001.

[30] D. P. Larson, L. Nonnenmacher, and C. F. M. Coimbra, "Day-ahead forecasting of solar power output from photovoltaic plants in the American Southwest," Renewable Energy, vol. 91, pp. 11-20, June 2016, doi: 10.1016/j.renene.2016.01.039.

[31] A. W. Aryaputera, D. Yang, and W. M. Walsh, "Day-Ahead Solar Irradiance Forecasting in a Tropical Environment," Journal of Solar Energy Engineering, vol. 137, no. 5, July 2015, doi: 10.1115/1.4030231.

[32] L. Hai, C. Zhang, Y. Y. Hong, Y. He, and S. Wen, "Day-ahead spatiotemporal solar irradiation forecasting using frequencybased hybrid principal component analysis and neural network," Applied Energy, vol. 247, pp. 389-402, Aug. 2019, doi: 10.1016/j.apenergy.2019.04.056. 


\section{BIOGRAPHIES OF AUTHORS}

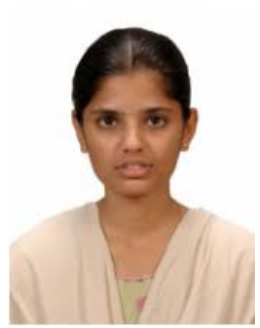

Sowkarthika Subramanian (D) $5 \mathrm{~B}$ ( $\mathrm{P}$ received the B.E degree in electrical engineering from $\mathrm{Ku}-$ maraguru College of Technology and M.E degree in power electronics and drives in PSG College of Technology. She is currently working as assistant professor in Government College of Technology. Her research interests include AI based power electronics applications to renewable energy systems. She can be contacted at email: sowkarthika@gct.ac.in.

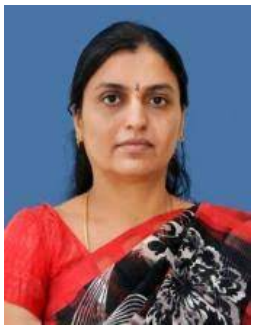

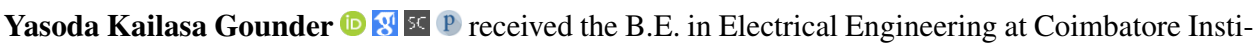
tute of Technology, Coimbatore, India, M.E. degree in Power Electronics and Drives from Alagappa Chettiar College Engineering and Technology, Karaikudi, India and Ph.D. from Anna University, Chennai, India. Currently, she is working as an assistant professor (senior grade) in Department of Electrical Engineering at Government College of Technology, Coimbatore, India. Her research interests are wind energy conversion systems, power electronics and micro grids. She can be contacted at email: yasoda@gct.ac.in.

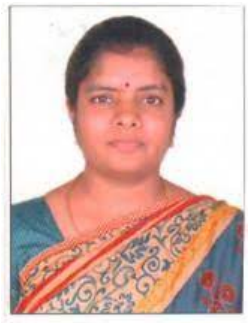

Sumathi Linganathan (D) Is P received the B.Tech degree in Information Technology from VLB Jannakiammal College of Engineering and Technology and M.E. in Computer Science and Engineering from Kumaraguru College of Technology. She worked as software engineer in Infosys Pvt. Ltd. from 2007 to 2008. She is currently working as assistant professor in Government College of Technology. Her research interests include Internet of Things, Machine Learning, and Cyber Security. She can be contacted at email: 1sumathi@gct.ac.in. 